\title{
Via-Hole Formation by Excimer Laser and Filling by Electro-Plating
}

\author{
Takashi Suzuki*, Toshio Tamura*, Atsushi Fujisaki*, Ryohei Yaegashi*, Toshimasa Urashima*, \\ Tadaaki Yamada*, Yohei Wakuda*,**, Satoshi Ando***, Akira Matsuno*** and Ichiro Koiwa** \\ *Noge Electric Industries Co., Ltd., 2-10-1, Fukuura, Kanazawa-ku, Yokohama 236-0004, Japan \\ **Kanto Gakuin Universcity, 1-50-1, Mutsuurahigashi, Kanazawa-ku, Yokohama 236-8501, Japan \\ ***Phoeton Corporation, 3050, Okada, Atsugi-shi, Kanagawa 243-0021, Japan
}

(Received July 28, 2008; accepted November 25, 2008)

\begin{abstract}
A multi-chip module (MCM) was fabricated using an excimer laser driller and electro-plating. This method contributes to the interconnection properties of the MCM. First, a second chip with a thickness of $50 \mu \mathrm{m}$ was mounted on a wafer that had been created by a wafer process, and polyimide with a thickness of about $100 \mu \mathrm{m}$ was applied by a spin-coater to cover the mounted chips. Two types of via-holes, with depths of 50 and $100 \mu \mathrm{m}$, were formed by the excimer laser to connect the wafer and mounted chip pads. The excimer laser driller with a micro-lens array formed two types of via with diameters of about $30 \mu \mathrm{m}$ simultaneously. Damage caused by the excimer laser irradiation was examined by direct laser irradiation of the FET transistor gate. Properties of the FET transistor did not change even after 500 pulses of $400 \mathrm{~mJ} /$ $\mathrm{cm}^{2}$ which is sufficient for via-hole formation. A micro-lens array was designed to shorten the via-hole formation time. After via-hole formation, a seed-layer of sputtered $\mathrm{Ti}$ and $\mathrm{Cu}$ films were necessary, followed by copper electro-deposition. Microscopy measurements confirmed that the seed-layers were uniformly formed from top to bottom of the via-hole. Generally the mixture of additives to complete the via-hole filling consists of brightener, leveler, and a suppressor. By controlling the leveler concentration, a via-hole with a diameter of $30 \mu \mathrm{m}$ and a depth of $100 \mu \mathrm{m}$ was perfectly filled by copper electroplating. In this way the multi-chip module was created by wafer-level chip size technologies (W-CSP) using an excimer laser driller.
\end{abstract}

Keywords: W-CSP, Excimer Laser, Micro-Lens Array, Electro-Plating

\section{Introduction}

In today's networking society; there is a strong trend to make all electronic devices smaller and multi-functional, so that they will be handy and wearable. Semiconductor technologies have been the main support in realizing this ubiquitous networking society, but now it is becoming seriously difficult to miniaturize semiconductors at a lower cost, because finer processes make costs much higher. In addition, physical limitations, such as gate oxide film thickness, are being reached. To address this situation, many researchers have actively investigated the system in package (SiP) concept, referring to a single package containing many chips that represents a complete system.

The three dimensional (3D) packaging technology is great importance in this development. Several kinds of 3D packages such as wafer-to-wafer bonding technologies[1] have been applied to things such as image sensor chips[2] and shared memory.[3] A chip-to-wafer technology has also been actively investigated.[4] This paper reports on SiP (chip-to-wafer) that has been fabricated using waferlevel chip size package (W-CSP) technologies. With this method, new chips are bonded on the wafer after it has been through a wafer manufacturing process (front end process and back end process). A polyimide resin is coated onto the chip and wafer, and via-holes are opened by an excimer laser combined with a micro-lens array. Finally the via-holes are filled by copper electro-plating.

One of the features of this method is the combination of excimer laser and micro-lens array, which shortens the time needed to form the via-holes. There are two types of via-holes: those that connect bonded chips are $50 \mu \mathrm{m}$ deep and those that connect wafers have depths of $100 \mu \mathrm{m}$. Another feature is the electro-plating technology used to simultaneously fill via-holes of different depths. 


\section{Experimental}

In this study, TEG chips (Phase0, Hitachi High-Technologies Co., LTD.) were used as the mounted chips; they were thinned to $50 \mu \mathrm{m}$ thickness and attached on the wafer with DAF tape. The polyimide resin was coated and via-holes were formed by MMLD (micro multi laser driller, Phoeton Corp.) which consists of the micro-lens array. Both titanium and copper were sputtered, and electroplating was performed to fill the via-holes and wiring on the surface of the polyimide.

Figure 1 presents the cross-sectional schematic models of SiP investigated in this study. Two chips are contained in a chip size package by the W-CSP method. Copper filling of via-holes with different depths is a necessary part of this method. Figure 2 is a schematic model of an MMLD

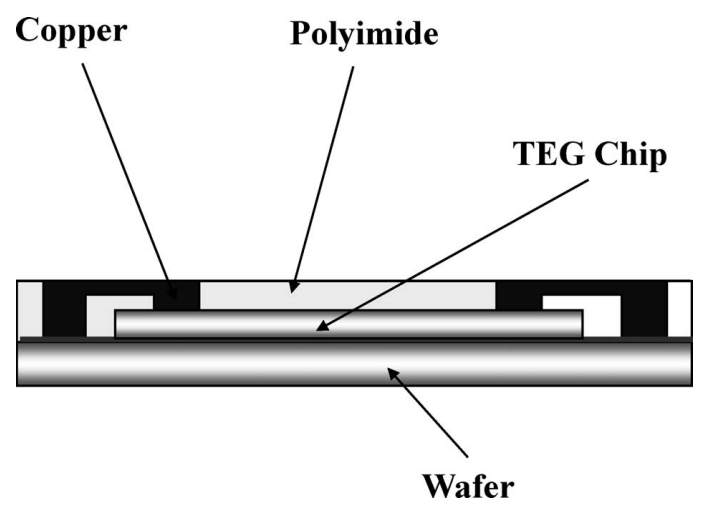

Fig. 1 Cross-sectional schematic model of SiP investigated in this study.

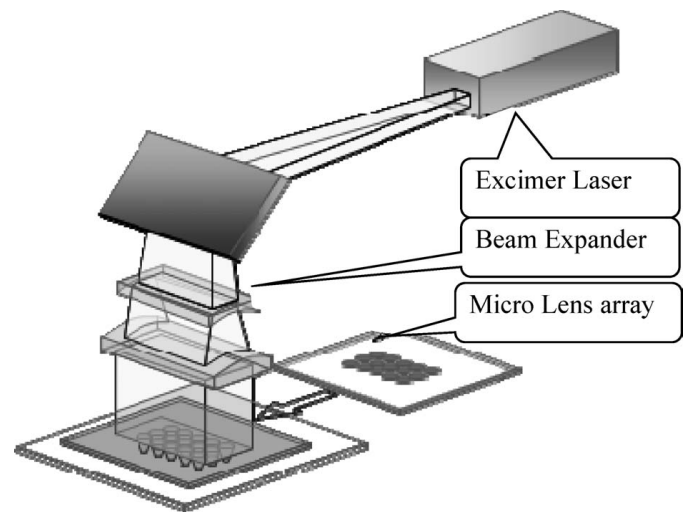

Fig. 2 Schematic model of combined excimer laser and micro-lens array.

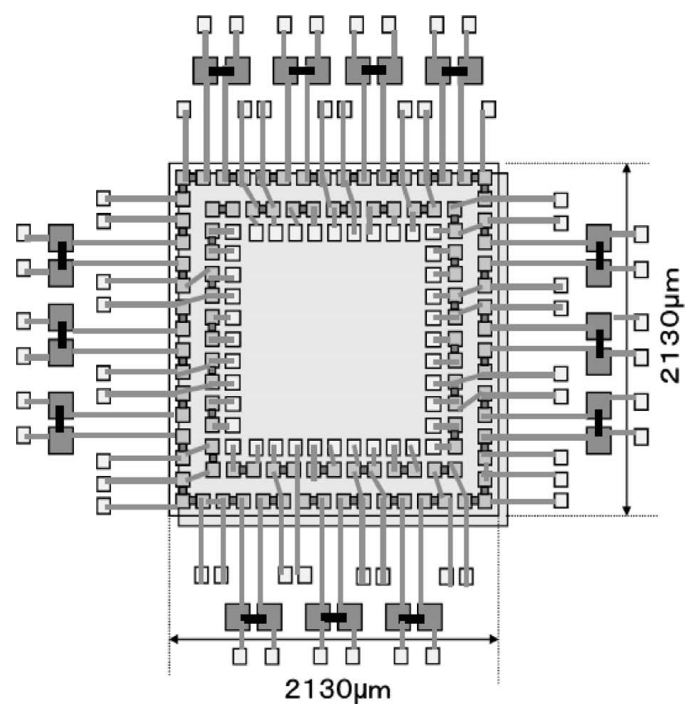

Fig. 3 Test pattern and chip pad location.

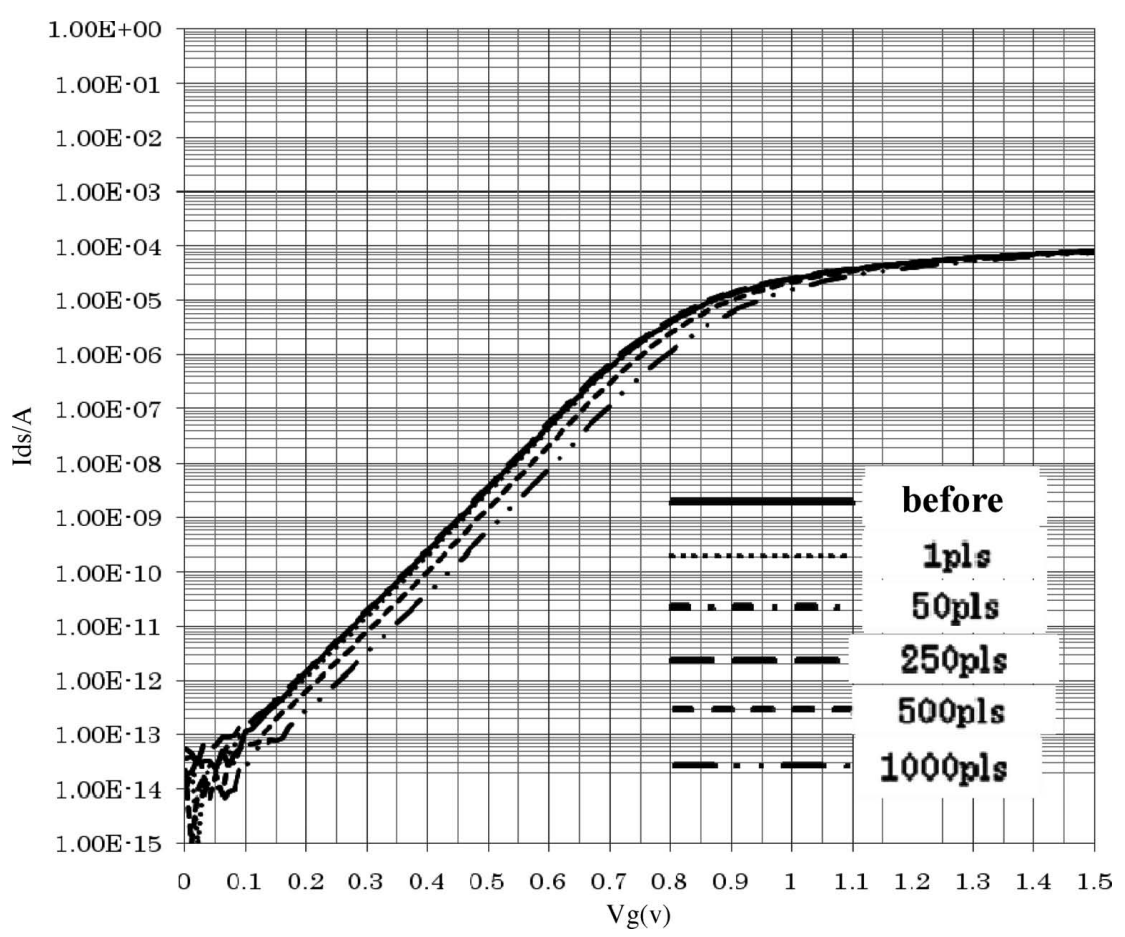

Fig. 4 Vg-Isd transistor curves for excimer laser irradiation. 
combined eximer laser and micro-lens array. The combination made it possible to form many via-holes at once. Figure 3 shows test patterns and pad locations of the TEG chip. The pad pitch of the TEG chip was $130 \mu \mathrm{m}$.

\section{Results and Discussion}

\subsection{Transistor damage from excimer laser irradiation}

As shown in Fig. 1, the irradiation times required to form via-holes with different depths, $50 \mu \mathrm{m}$ and $100 \mu \mathrm{m}$, are different. The irradiation time needed for shallower viaholes was much shorter than that for deeper ones, and the excimer laser continued irradiating until all via-holes were formed. The effects of excimer laser irradiation should be researched and irradiation conditions should be limited to the range within which there is no change to the transistor characteristics.

Figure 3 demonstrates the effect of laser irradiation on the relationship between gate voltage, $V_{g}$, and source-drain current, $\mathrm{I}_{\mathrm{ds}}$. The irradiated pad was directly connected to the gate electrodes. The transistor length/width, L/W, was $0.8 / 9.75 \mu \mathrm{m}$ and the thickness of the gate insulator oxide film was $15 \mathrm{~nm}$. A voltage of $1 \mathrm{~V}$ was applied between the source and drain. As seen in Fig. 4, no change of $V_{g}-I_{d s}$

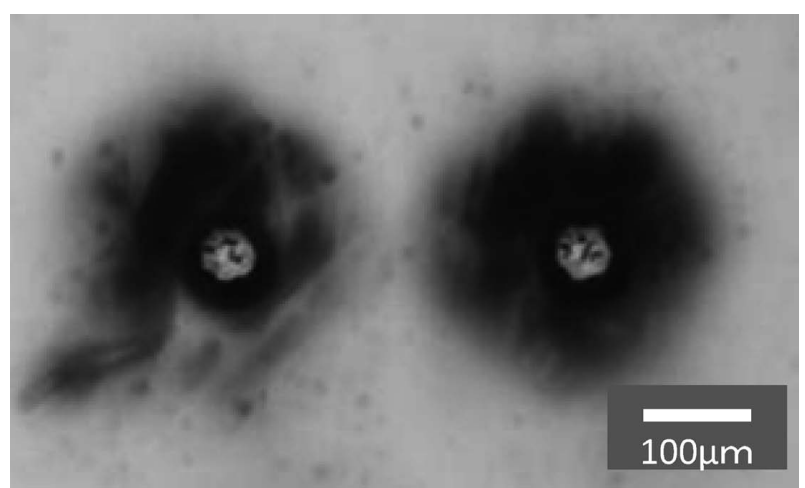

Fig. 5 Surface view of the via-holes. curves were observed when the pulse number was changed to $0,1,50,250,500$ and 1,000 , while pulse energy was kept at $500 \mathrm{~mJ} / \mathrm{cm}^{2}$.

\subsection{Via-hole shape formed by MMLD}

Figure 5 presents an overview of the via-holes, which were formed by 240 pulses with energy of $240 \mathrm{~mJ} / \mathrm{cm}^{2}$. Surface cleaning was performed by plasma ashing using $\mathrm{CF}_{4}$ gas. Figures 6(a) and (b) are SEM micrographs of the PI film surface after excimer laser irradiation and after ashing. It is shown that ashing using $\mathrm{CF}_{4}$ gas effectively

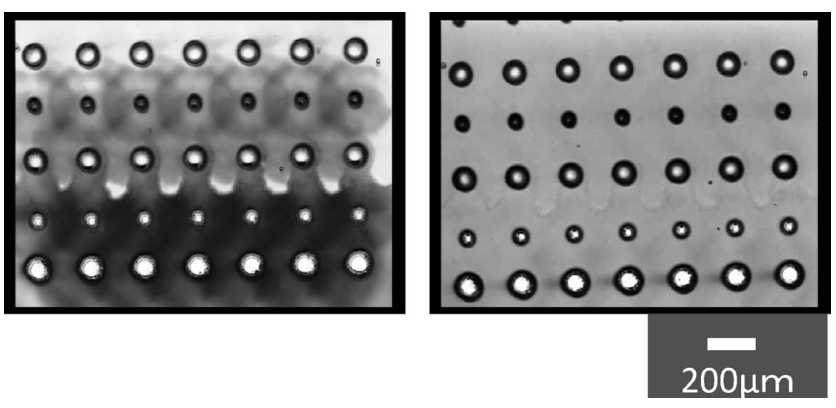
a) After laser irradiation.
b) After plasma ashing with $\mathrm{CF}_{4}$ gas.

Fig. 6 Surface cleaning after laser irradiation.

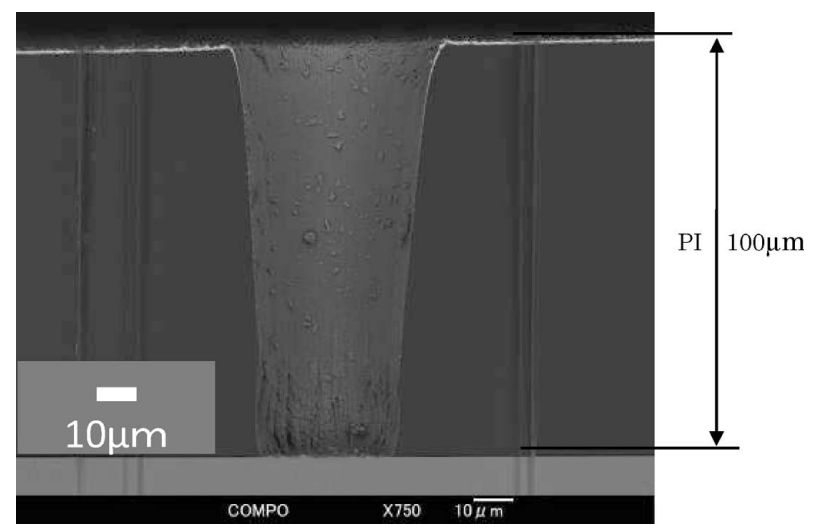

Fig. 7 Cross section of via-hole.

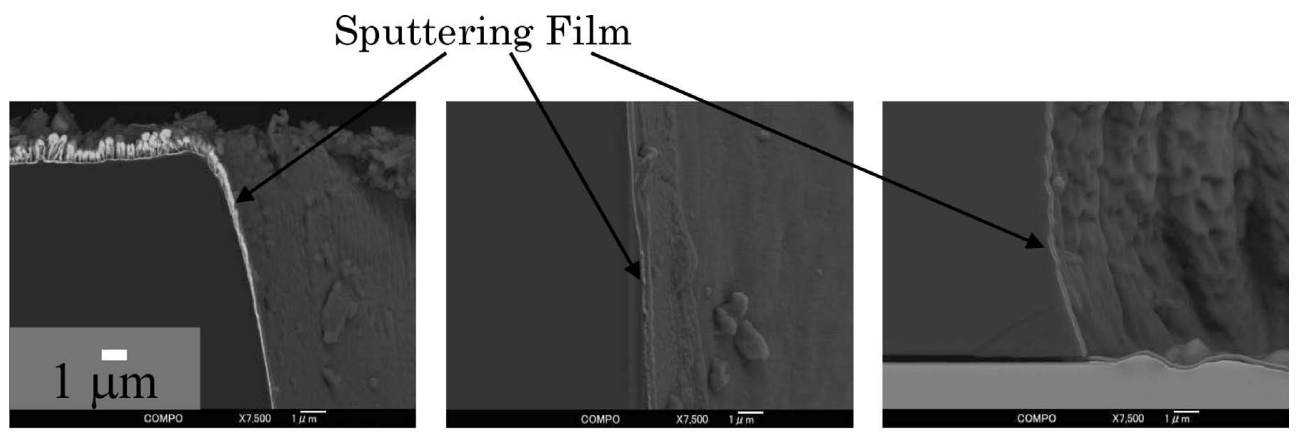
(a) Top parts of via-hole
(b) Side wall of via-hole
(c) Bottom part of via-hole

Fig. 8 SEM images of micrographs of via-hole. 


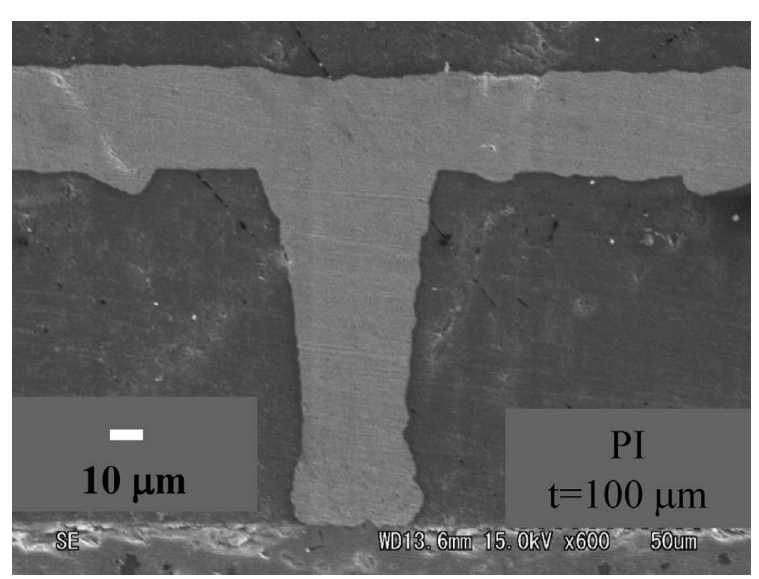

Fig. 9 Cross-sectional SEM micrographs after via-hole filling with $\mathrm{Cu}$ plating formed by excimer laser irradiation.

cleaned the PI film surface after excimer laser irradiation.

\subsection{Film formation inside via-hole by sputtering}

Figures 7 and 8(a), (b) and (c) are cross-sectional views of via-holes after sputtering titanium to $0.15 \mu \mathrm{m}$ thickness and copper to $0.3 \mu \mathrm{m}$.

A highly uniform sputtered film is necessary in order to proceed to via-hole filling. These figures confirm that sputtered film was formed from top to bottom of the via-hole. The top diameter of the via-hole was $50 \mu \mathrm{m}$ and the bottom diameter was $30 \mu \mathrm{m}$.

From Figs. 7 and 8(a), (b) and (c), we can see that the thickness of the sputtered film was $10 \mathrm{~nm}$ on the side walls and $20 \mathrm{~nm}$ at the bottom of the via-hole. As mentioned above, the sputtered thickness was $450 \mathrm{~nm}$, so the thickness on the side walls and the bottom were relatively thin, but the sputtered films were continuously formed.

Figure 9 is a cross-sectional SEM micrograph of the viahole after it was filled with $\mathrm{Cu}$ plating formed by the excimer laser irradiation. The via-hole was completely filled by copper plating and the surface of the plated film was flat. Therefore, this method has high potential to produce high density via-holes with diameters less than $30 \mu \mathrm{m}$.

\section{Conclusion}

Via-hole formation by MMLD and filling using electroplating were investigated and the following results were obtained.

(1) Two types of via having different depths, 50 and $100 \mu \mathrm{m}$, were formed by the MMLD to connect pads between the wafer pad and mounted chip pad.

(2) The MMLD formed two types of via with a diameter of about $30 \mu \mathrm{m}$ simultaneously.
(3) Damage from the excimer laser irradiation was examined by direct laser irradiation of the gate of the FET transistor. Properties of the FET transistor did not change even after 500 pulses of 400 $\mathrm{mJ} / \mathrm{cm}^{2}$, which was enough for via-hole formation.

(4) A micro-lens array was designed to shorten the via-hole formation time. After via-hole formation, a seed-layer of sputtered $\mathrm{Ti}$ and $\mathrm{Cu}$ films was necessary, followed by copper electro-deposition.

(5) Microscopy measurements confirmed the seedlayer was uniformly formed from top to bottom of the via-hole.

(6) Via-holes with a $30 \mu \mathrm{m}$ diameter and $100 \mu \mathrm{m}$ depth have been perfectly filled by copper electroplating . Therefore this study has shown it is possible to form a multi-chip module using W-CSP techniques with an excimer laser.

\section{Acknowledgements}

This work was supported by "subsidies for projects to promote the enhancement of manufacturing technology for small and medium enterprises (SMEs)" project grant by Organization for Small \& Medium Enterprises and Regional Innovation, JAPAN (SMRJ).

\section{References}

[1] T. Matsumoto, M. Satto, K. Sakuma, H. Kurino, N. Miyakawa, H. Itani and M. Koyanagi, "New Threedimensional Wafer Bonding Technology Using the Adhesive Injection Method”, Jpn. J. Appl. Phys., Vol. 37, pp. 1217-1221, 1998.

[2] H. Kurino, K. W. Lee, T. Nakamura, K. Sakuma, K. T. Park, H. Shimazutsu, K. Y. Kim, K. Inamura and M. Koyanagi, "Intelligent Image Sensor Chip with Three Dimensional Structure”, IEEE International Electron Device Meeting (IEDM), Technical Digest, pp. 879882, 1999.

[3] K. W. Lee, T. Nakamura, T. Ono, Y. Yamada, T. Mizukusa, H. Hashimoto, K. T. Park, H. Kurino and M. Koyanagi, "Three-Dimensional Shared Memory Fabricated Using Wafer Stacking Technology”, IEEE International Electron Devices Meeting (IEDM) Technical Digest, pp. 165-168, 2000.

[4] A. Klumpp, R. Merkel, P. Ramm, J. Weber and R. Wieland, "Vertical System Integration by Using InterChip Vias and Solid-Liquid Interdiffusion Bonding", Jpn. J. Appl. Phys., Vol. 43, pp. L829-L830, 2004. 